\title{
Brain Tumour Detection using Fine-Tuning Mechanism for Magnetic Resonance Imaging
}

\author{
Mohammed S. H. Al-Tamimi ${ }^{1}$, Nur Hafizah Ghazali ${ }^{2,}$, Norfadila Mahrom ${ }^{2}$, Nurulhuda Ghazali ${ }^{3}$, and Ghazali \\ Sulong ${ }^{4,5}$ \\ ${ }^{1}$ University of Baghdad, College of Science, Department of Computer Science, Baghdad, Iraq \\ ${ }^{2}$ Universiti Malaysia Perlis, School of Computer and Communication Engineering, Perlis, Malaysia \\ ${ }^{3}$ Universiti Teknologi Mara, Faculty of Computer and Mathematic Sciences, Melaka, Malaysia \\ ${ }^{4}$ Universiti Teknologi Malaysia, Faculty of Computing, Johor, Malaysia \\ ${ }^{5}$ Universiti Malaysia Terengganu, School of Informatics and Applied Mathematics, Terengganu, Malaysia
}

\begin{abstract}
In this paper, new brain tumour detection method is discovered whereby the normal slices are disassembled from the abnormal ones. Three main phases are deployed including the extraction of the cerebral tissue, the detection of abnormal block and the mechanism of fine-tuning and finally the detection of abnormal slice according to the detected abnormal blocks. Through experimental tests, progress made by the suggested means is assessed and verified. As a result, in terms of qualitative assessment, it is found that the performance of proposed method is satisfactory and may contribute to the development of reliable MRI brain tumour diagnosis and treatments.
\end{abstract}

\section{Introduction}

In recent years, tumor incidence has become rapidly increasing and this gives cause for concern. A tumor exist when new cells in an organism growth abnormally. These new cells grow very quickly compared to normal cells. Upon growing, these cells can cause damage to adjacent structures or cells and might be fatal. Brain is the most vital organ in human being and therefore brain tumor has always been taken serious by medical practice around the world in order to detect and treat the disease as early as possible. To ensure an accurate diagnosis of the brain tumor, an imaging technique is used to measure the volume of the tumor. A common imaging technique that has been widely used and accepted for the detection of brain tumor image is Magnetic Resonance Imaging (MRI). MRI is a type of scan that is used in radiology to gather pictures of the anatomy and the physiological processes in an organism. The process involved the usage of strong magnetic fields, radio waves and field gradients to yield organ images. From the image, doctors will evaluate and diagnose the condition of the tumor and come out with possible treatments. Most of the times, distinguishing between normal slice and abnormal slice of MRI images are quite challenging. Thus, a proper method is crucial to identify and extract the true location of the tumor. Experiments were conducted on a set of digitized MRI images from three types of dataset that were obtained from Internet Brain Segmentation Repository (IBSR) and multimodal Brain Tumor Segmentation (BRATS) challenge from 1st International Conference on Medical Image Computing and Computer Assisted Intervention (MICCAI 2012).

\footnotetext{
Corresponding author: hafizahghazali@unimap.edu.my
}

The process of detecting abnormality in MRI brain slices is somewhat a thorough, extensive and timeconsuming clinical procedure (Singh and Kaur 2012; Kumari 2013; Salankar and Bora 2014). This circumstance is explained by two underpinning factors : (1) homogeneity that occurs among healthy tissues and cancerous cells which are unable to be figured out by naked eyes and some machines, and (2) the presence of humongous amount of slices depending on the type and degree of illnesses (Selvaraj et al. 2007; Abdullah et al. 2011; Salankar and Bora 2014). Due to these reasons, several trials have been conducted in order to automate the procedure but they were all below the expectations thus entail future improvements. In addition, another glaring matter lies on locating a tumour or cancerous cell present in the abnormal slice where no research has been conducted on this at present. Hence, there is a need for a solution to the problem which will not only equip doctors with a cutting-edge but also to provide a fruitful performance of the following processes which are segmentation, visualization and volume estimation of tumours.

\section{Methodology}

This part practices the latest procedures to separate the normal slices from the abnormal ones in the brain MR images. There are four steps involved for the proposed method: (1) Extraction of cerebral tissues, (2) Determination of the maximum value for separating the blocks in normal and abnormal kinds, (3) Detection of abnormal blocks via threshold gained in step 2, (4) 
Output - identified abnormal slices together with the tumour region.

The threshold value is needed in the process of differentiation of the abnormal MRI slices from the normal ones. Previous works of threshold method used watershed and histogram analysis (Mustaqeem et al. 2012), Inter-Hemisphere Fissure (I-HF) and symmetrical nature threshold (Somasundaram and Kalaiselvi 2010), and Otsu's threshold (Amrutal et al. 2010). However, in this work, the maximum threshold value is gained from statistical calculation which takes into account the feature parameters including mean, energy and entropy. Once the abnormal blocks inside the slices are detected, the abnormal MRI slices can be gained hence it is fundamental to ascertain the abnormal blocks and refine them in getting the accurate abnormal slice.

There are three subsections present in this section. In detail, Subsection 2.1 explains the method of extracting cerebral tissues. While Subsection 2.2 presents the determining method in gaining the optimal threshold value and subsection 2.3 explains the detection process of tumor block abnormality in the MRI.

\subsection{Extraction of Cerebral Tissues}

The extraction of the cerebral tissues has four main steps. (1) Image binarization where the transformation of gray scale images $(0-255)$ into binary image through two values of zero and one (Somasundaram and Kalavathi 2011; Jain 2013). This is conducted in order to expel the non-cerebral where in binarization, the threshold level is identified as being either white or black. In this case, Otsu's automatic thresholding algorithm is regarded as a benchmark (Kotte et.al 2016; Hamamci et al. 2012; Zhang et al. 2012).

After that, the determination of the Largest Connected Component or LCC is conducted by locating the component of the largest non-cerebral tissue region through several numbers of connected components inclusive of skull, bone, eyeballs and dura within that slice (Park and Lee 2009; Somasundaram and Kalaiselvi 2011). The proposed technique then separates MRI slice into joined components which combine all the connected white pixels according to its connectivity. In each of these components, 8-side connectivity is used to measure the adjacencies among pixels and they are later labelled orderly using numbers. Similarly labelled pixels represents a component that is extracted through the image to segment each component individually. After all joined components are identified, overlapping might occur where the extension of a component region into another constituent area. As a result, the proposed technique will again locate the connected parts and consider only constituent that possesses the larger area based on pixels. Once all joined components in the MRI slice image are located, the biggest constituents with pixels in every labelled area are found by one loop.
Once the LCC is detected through the previous stage, a binary mask which is based on the LCC pixels is established in the same size as the binary MRI slice. They are then labelled as black and the others as white.

The binary mask for a brain is located through earlier steps where the cerebral tissues are extracted through simple bitwise operation "AND" between the actual MRI slice and the binary mask. It protected the pixels of cerebral tissues corresponding to translucent areas and concurrently restructured the pixels of the masked overlapping image bits with zeros. Fig. 1 displays the binary mask operation of this MRI slice.

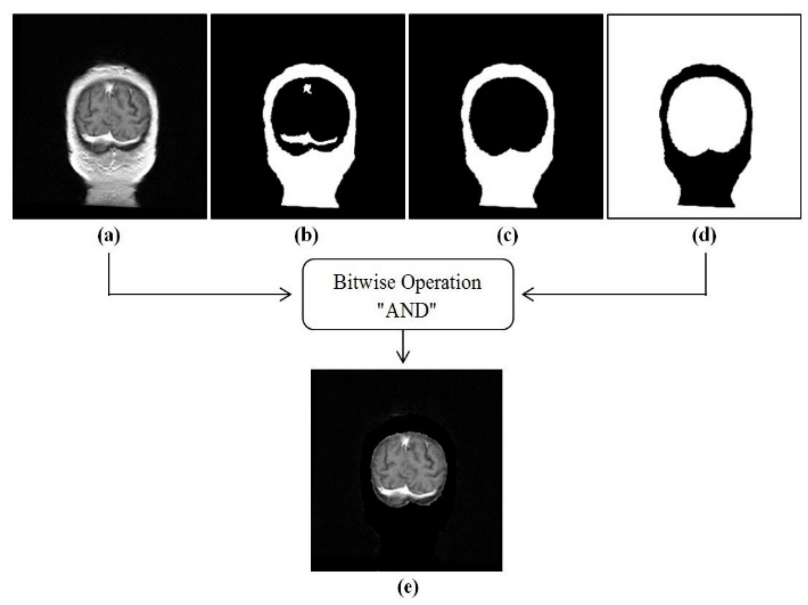

Fig. 1. The cerebral tissue extraction of MR image, (a) Original slice, (b) Binary image with three joined components (white regions), (c) The LCC (the largest white region), (d) The LCC mask (black region), and (e) Extracted cerebral tissue

\subsection{Determination of Thresholds}

Before the detection of abnormality took place, optimal threshold values are identified statistically through three parameters which are mean, energy and entropy. Then, each MR image that has a set of slices are grouped into non overlapping of $8 \times 8$ pixels that is regarded as optimal due to its empirical state that is determined by experiments through several block sizes ranging from $4 \times 4$ to $16 \times 16$ pixels. After that, for each block, the stated parameters are extracted to identify three threshold values which are the Mean-threshold $\left(\mathrm{T}_{1}\right)$, Energythreshold $\left(\mathrm{T}_{2}\right)$, and Entropy-threshold $\left(\mathrm{T}_{3}\right)$. Statistically, the characteristics are calculated using equation (1), (2) and (3) respectively.

$$
\operatorname{Mean}(\mu)=\frac{\sum_{x=1}^{w} \sum_{y=1}^{w} I(x, y)}{w \times w}
$$

$$
\begin{aligned}
& \text { Energy }(e)=\frac{1}{w \times w} \sum_{x=1}^{w} \sum_{y=1}^{N} I^{2}(x, y) \\
& \text { Entropy }=\frac{1}{w \times w} \sum_{x=1}^{w} \sum_{y=1}^{w} I(x, y)(-\ln l(x, y))
\end{aligned}
$$

where $(\mathrm{w}=8)$ is the block size. A correlation between these characteristics and their distribution in the MRI 
slice is identified in order to gain the optimal threshold values.

\subsection{Detection of Abnormal Blocks}

The identification of abnormal blocks within each MRI slice is the conducted after the threshold values have been successfully determined. It is clear that if abnormal blocks are present in a particular slice, it will be regarded as abnormal where $T_{1}, T_{2}$ and $T_{3}$, are used to locate the abnormal blocks. A block is identified as abnormal if and only if all the rules are satisfied where the rule is shown in Fig. 2.

if Mean $>T_{1}$ then if Energy $>T_{2}$ then

if Entropy $>T_{3}$ then

Block is Tumour

else

Block is Non-Tumour

Fig. 2. Abnormal block detection rules

Nonetheless, the achieved result might not be $100 \%$ accurate as some blocks of tumours might be captured as the non-tumour part. This somehow influences the results of abnormality detection of the slice hence a refined tuning procedure is suggested in order to check, validate and enhance the initial normal block. The tuning procedure is done through three types of $3 \times 3$ block masks which are preceding, current, and succeeding block mask as shown in Fig. 3.

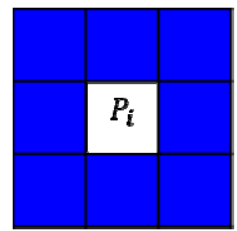

(a)

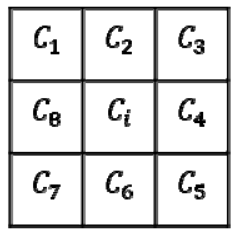

(b)

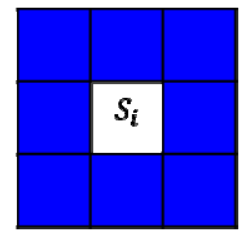

(c)
Fig. 3. Three types of block masks with size $(3 \times 3)$ called (a) Preceding mask centred at $\mathrm{P}_{\mathrm{i}}$, (b) Current mask centred at $\mathrm{C}_{\mathrm{i}}$, and (c) Succeeding mask centred at $\mathrm{S}_{\mathrm{i}}$. Blue colour indicates ignored blocks.

This procedure is done by scanning MRI slice blockby-block starting from the top-left to the right-bottom corners through the Current Block Mask starting from the top-left corner and moves a column to another till the last one. After that. it moves down one row and starts again from the first column until it reaches the last column and so on so forth until it reaches the rightbottom corner. The scanned slice is then labelled as tumour slice if it has at least a tumour block. This mechanism does not only identify or locate the tumour slice, it also shows location and size (in terms of block) of tumour regions which is also known as Region of Interest (ROI).

\section{Experimental Results and Discussion}

Using publicly available IBSR (10Normals_T1), IBSR

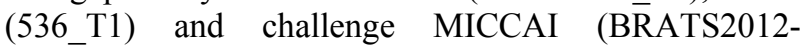
BRATS-1) datasets comprised of 4567 MRI slices of 35 patients out of which 25 are cancerous and 10 noncancerous, a series of experimentations are handled where qualitative measurements are based on visual human inspections.

Abnormality detection of MRI slices is conducted by doing a sequence of experiments to assess the performance of the proposed abnormal MRI slice detection inclusive of (1) Cerebral tissue extraction, (2) Abnormal block detection (before and after the finetuning mechanism), and (3) The abnormal slice detection.

\subsection{Results of Cerebral Tissue Extraction}

In this stage, qualitative evaluation on various random MR slice images gained from the selected dataset is conducted. Fig. 4 depicts an original MRI slice containing both cerebral and non-cerebral tissues.

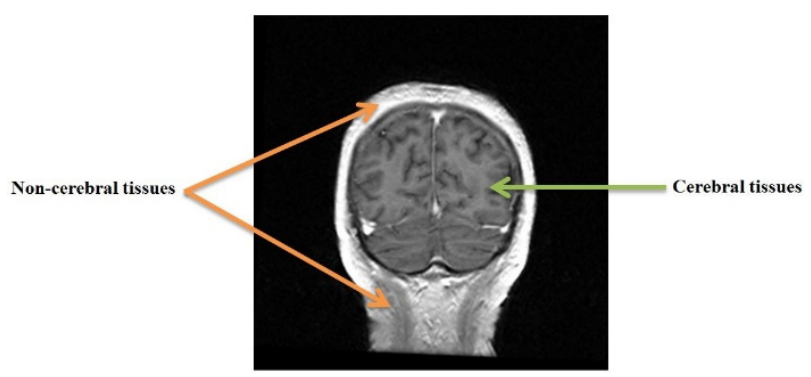

Fig. 4. The original slice showing cerebral and non-cerebral tissues

In order to extract the cerebral tissues via bitwise operations between the original MRI slice with the binary mask of the LLC, subsection 2.1 is conducted. Hence, only the cerebral tissues are maintained in the MRI slice when this mask is done, as illustrated in Fig. 5 .

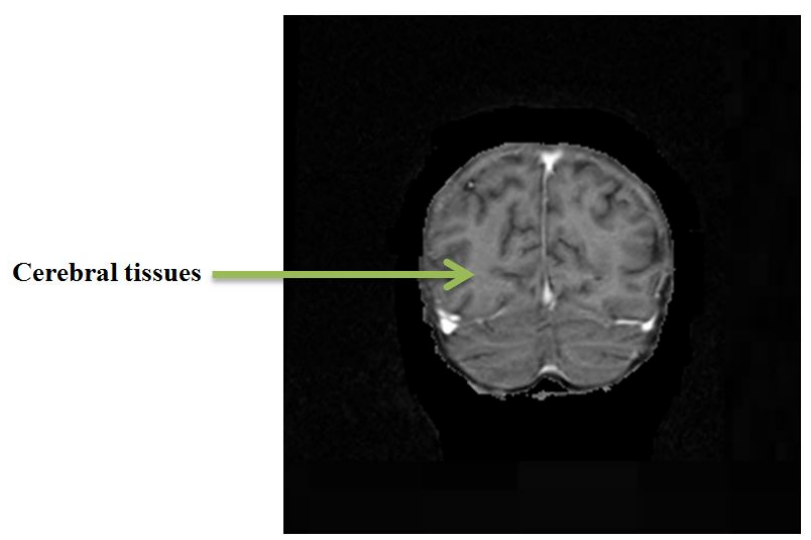

Fig. 5. Result on cerebral tissue extraction 
Fig. 6 shows the experimental results for cerebral tissues of MRI brain slices obtained from IBSR (536_T1) dataset using the proposed method. The left column is the original MRI slice image while the right column is the cerebral tissues gained from the MRI slice.
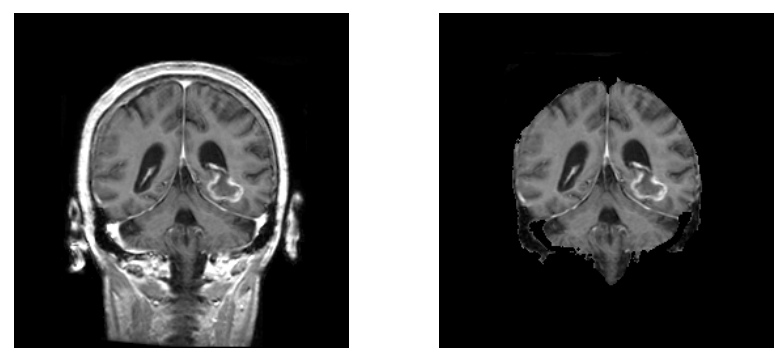

Slice 22
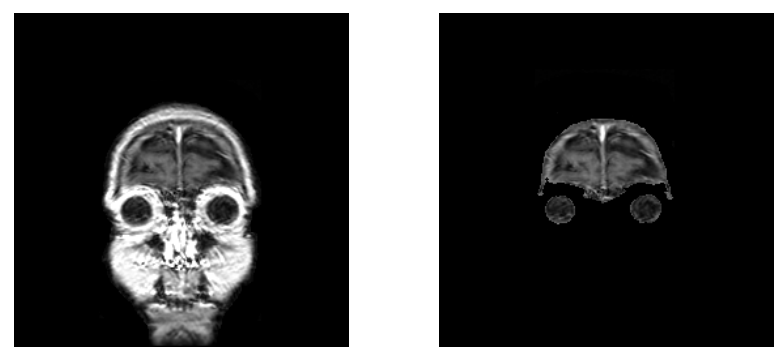

Slice 55

Fig. 6 Results of cerebral tissue extraction from IBSR (536_T1) dataset

The relevance of the proposed method is clearly disclosed by the thorough visual inspection of these extracted cerebral tissue MRI slices. The extraction mechanisms are able of removing all parts of noncerebral tissues with high accuracy, including skull, bone, eyeballs and the dura.

\subsection{Results of the Abnormal Block Detection Before Fine-Tuning}

The extracted cerebral tissues are very significant for ROI tumour recognition through non-overlapping block division. Three characteristics such as mean, energy and entropy are obtained from each block where relationship between these features and their distribution in the MRI slice are evaluated using three dimensional (3D) graphs. By implementing Otsu's automatic thresholding algorithm, three distinctive thresholds are developed to represent these extracted features. The Otsu's algorithm is considered as the benchmark in this field. The performance of the current method is assessed using visual inspection mechanism. Fig. 7, 8, and 9 illustrate the experimental results of the block classification using three datasets, where the left column shows the original MRI slices image, the centre is the $(8 \times 8)$ block division using the proposed method and the right column is block detection result where the blue colour represents the normal region (non-tumour area) and the other blocks signifies the abnormal area (tumour area).
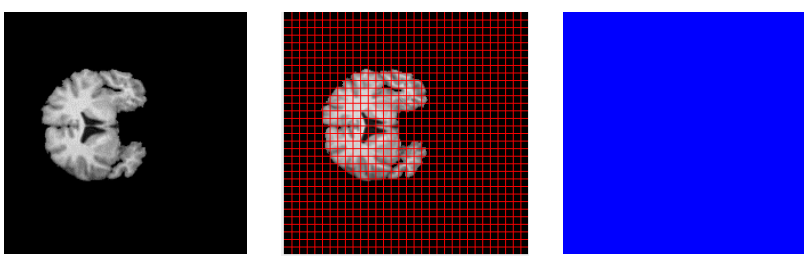

Fig. 7 Abnormal block detection result of MRI slice of IBSR (10Normals_T1) dataset
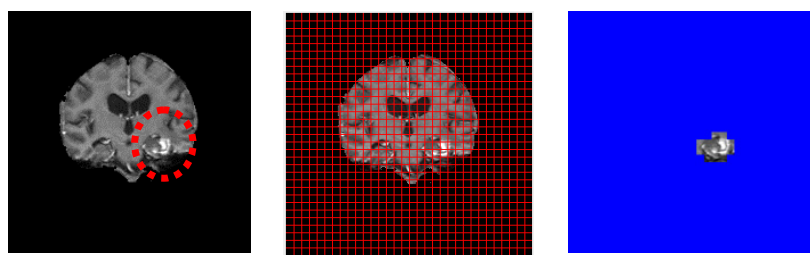

Fig. 8 Abnormal block detection result of MRI slice of IBSR (536_T1) dataset where the tumour initiated by a red circle
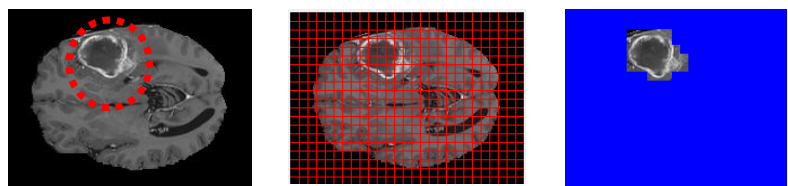

Fig. 9 Abnormal block detection result of MRI slice of MICCAI (BRATS2012-BRAST-1) dataset where the tumour initiated by a red circle

Based on the visual inspection of these slices, it is clearly discovered that the proposed abnormal block detection method, in terms of the brain tumour region, resulted in reasonably excellent result. Nevertheless, several misclassified results were found using the proposed method. Fig. 10, 11, and 12 depict the experimental results for the three datasets, where the proposed method misclassified the block. The term misclassified is defined as "a questioned block is supposed to be tumour but the method labelled it as nontumour, or vice versa". Due to this, some blocks of tumour region are identified as non-tumour (yellow circle) and numerous normal blocks happened to be the part of the tumour region which is represented by red circle.
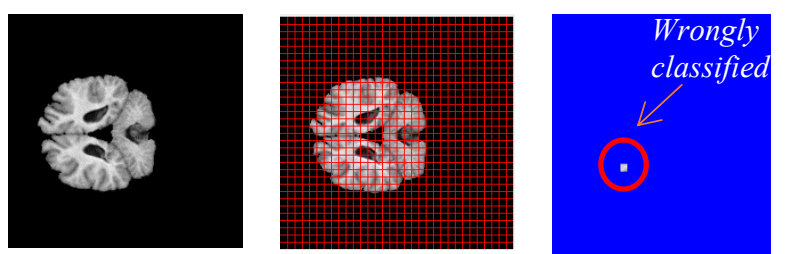

Fig. 10 Misclassified block of MRI slice of IBSR (10Normals_T1) dataset without any tumour
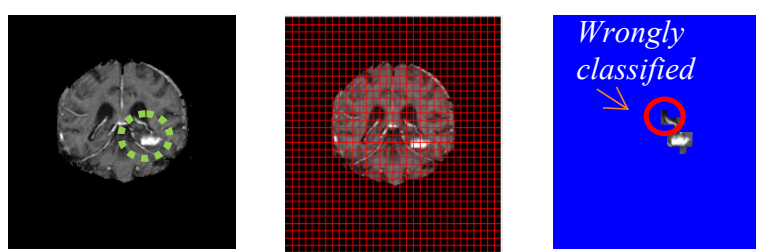

Fig. 11 Misclassified block of MRI slice of IBSR (536 T1) dataset with tumour marked via green circle 

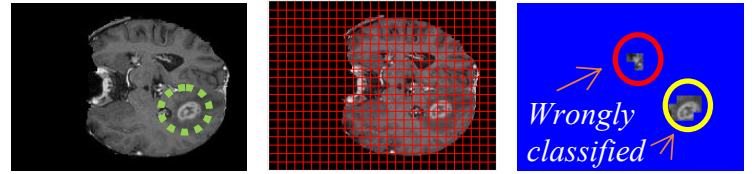

Fig. 12 Misclassified block of MRI slice of MICCAI (BRATS2012-BRAST-1) dataset with tumour initiated by circle

\subsection{Results of the Abnormal Block Detection with Fine-Tuning}

It is very clear that the inaccurate identification of the tumour region greatly influences the results of slice abnormality detection. This matter is resolved by applying sets of rules based on the neighbouring blocks. First, three types of $(3 \times 3)$ blocks mask namely "Preceding, Current, and Succeeding Blocks Mask" block-by-block are adopted in order to perform scannings of the MR slice image from top to bottom and from left to right direction. Next, all the neighbouring blocks surrounding a centre block in the "Current Block Mask" as well as the centred block of "Preceding, and Succeeding Blocks Mask" are chosen in the final decision of the block classification method. Accurate result is obtained by applying all previously mentioned rules (Subsection 2.3), where the occurrence of inexact classification blocks is then modified through the proposed method. This process is vital as the reliability of abnormality detection is primarily dependent on the accuracy of this mechanism. (a)

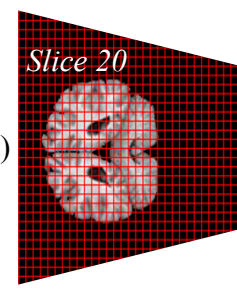

(b)

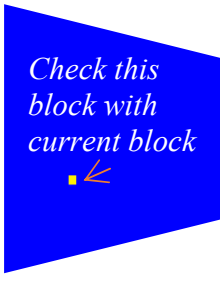

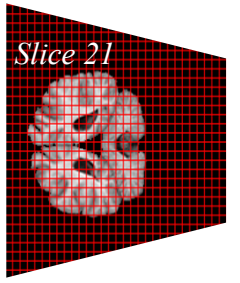
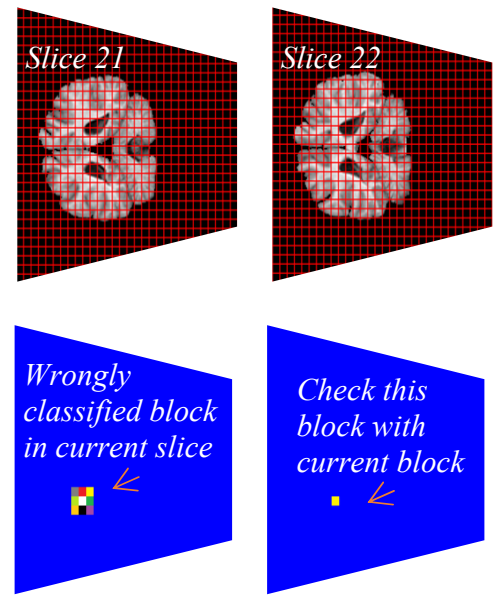

(c)

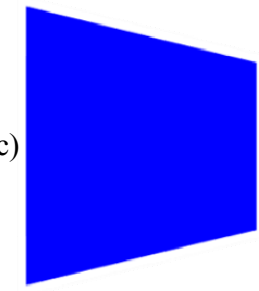

Fig. 13 A fine-tuning result of slice 21 of IBSR (10Normal T1) dataset: (a) The original non-tumour slice 21,

(b) Fine-tuning of the misclassified block of current slice, and

(c) Final result of the questioned block

Now the rules are deployed to correct the wrong classifications. Fig. 13, 14, and 15 depict the corrected slices after taking out the inaccuracy. The first, second and third columns show the preceding slice, the current slice, and the succeeding slice, respectively. (a)

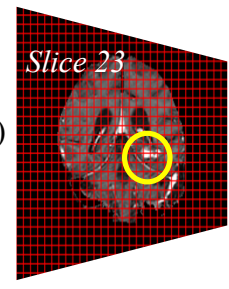

(b)

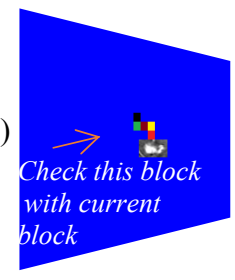

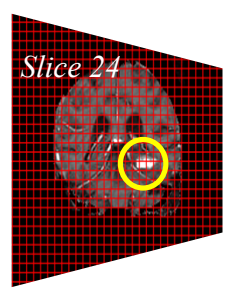

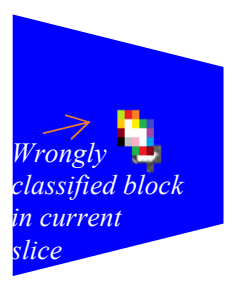

(c)

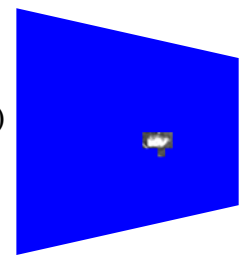

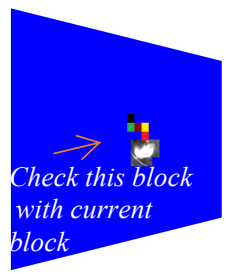
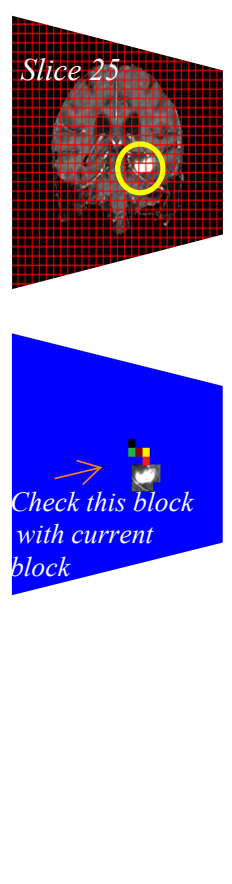

Fig. 14 A fine-tuning result of five tumour blocks of slice 24 from IBSR (536 T1) dataset: (a) The original tumour slice 21, (b) Fine-tuning of the misclassified block of current slice, and (c) Final result of the questioned blocks

It is prominent that from the above findings, the proposed fine-tuning procedure has successfully solved the misclassification issues and enhanced the accuracy of the tumour block detection. In general, the abnormal region posseses all the important characteristics of a tumour and any incorrectness in the identifications of this region has severe consequences with regards to anomaly detection and treatment. Through the proposed abnormal block detection method coupled with the finetuning procedure, the tumour region is perfectly selected (i.e. ROI) for better tumour slice detection and segmentation mechanism. As a whole, in terms of the qualitative assessment, the performance of all parts of the current methods, including cerebral tissue extraction, abnormality block classification and fine-tuning procedure are of a satisfactory level.

\section{Conclusion}

Through a new detection procedure for MRI brain abnormality presented in this paper, a set of preprocessing measures is executed with the MR images containing non-cerebral tissue extraction. These measures included the binarization of MRI slices, followed by their LCC detection. Then, a binary mask is gained from the extracted LCC and bitwise operations are applied between the original slice and the binary mask to extract the cerebral tissue from the MR image. As a result, three distinctive thresholds, which are based 
on texture features: mean, energy and entropy, are obtained automatically thus permits accurate separation of the MRI slice into normal and abnormal ones. Nevertheless, the abnormality detection showed some normal blocks which are placed incorrectly as abnormal and vice versa. This issue is surmounted by applying the fine-tuning mechanism of which the MRI slice abnormality detection is finally achieved by selecting the abnormal slices along its tumour region. The presented mechanism is suitable to be clinically practiced in the procedures of treating cancers including surgery, cancer drugs, chemotherapy and radiotherapy.

\section{Acknowledgement}

The authors would like to thank Universiti Malaysia Perlis for providing the facilities and financial support (Short Term Grant No. 9001-00567) to conduct this research.

\section{References}

1. D. Singh, K. Kaur, (2012). International Journal of Engineering and Advanced Technology (IJEAT), Classification of Abnormalities in Brain MRI Images Using GLCM , PCA and SVM., vol.1, no.6, pp.243-248.

2. R. Kumari, (2013). SVM Classification an Approach on Detecting Abnormality in Brain MRI Images. International Journal of Engineering Research and Applications (IJERA), vol.3, no.4, pp.1686-1690.

3. S. S. Salankar, V. R. Bora, (2014). MRI Brain Cancer Classification Using Support Vector Machine. Electrical, Electronics and Computer Science (SCEECS), IEEE Students' Conference. pp. $1-6$.

4. A. Mustaqeem, A. Javed, T. Fatima, (2012). An Efficient Brain Tumor Detection Algorithm Using Watershed and Thresholding Based Segmentation. International Journal of Image, Graphics and Signal Processing, vol.4, no.10, pp.34-39.

5. K. Somasundaram, T. Kalaiselvi, (2010). Automatic Detection of Brain Tumor from MRI Scans Using Maxima Transform. UGC Sponsored National Conference on Image Processing-NCIMP. pp. 136141.

6. A. Amrutal, A. Gole, Y. Karunakar, (2010). A Systematic Algorithm for 3-D Reconstruction of MRI based Brain Tumors using Morphological Operators and Bicubic Interpolation. Computer Technology and Development (ICCTD), $2^{\text {nd }}$ International Conference on. IEEE. pp.305-309.

7. H. Selvaraj, S. T. Selvi, D. Selvathi, L. Gewali, (2007). Brain MRI Slices Classification Using Least Squares Support Vector Machine. International Journal of Intelligent Computing in Medical
Sciences and Image Processing, vol.1, no.1, pp.2133.

8. N. Abdullah, U. K. Ngah, S. A. Aziz, (2011). Image Classification of Brain MRI Using Support Vector Machine. Imaging Systems and Techniques (IST), IEEE International Conference, pp. 242-247.

9. K. Somasundaram, P. Kalavathi, (2011). Medical Image Binarization Using Square Wave Representation. First Edition. Springer-Verlag Berlin Heidelberg.

10. S. Jain, (2013). Brain Cancer Classification Using GLCM Based Feature Extraction in Artificial Neural Network. International Journal of Computer Science and Engineering Technology, vol.4, no.7, pp.966-970.

11. S. Kotte, P. R. Kumar, S. K. Injeti, (2016). An Efficience Approach for Optimal Multilevel Thresholding Selection for Gray Scale Images Based on Improved Differential Search Algorithm. Ain Shams Engineering Journal.

12. A. Hamamci, G. Unal, (2012). Multimodal Brain Tumor Segmentation Using The "Tumor-Cut" Method on The BraTS Dataset. Process MICCAIBRATS (Multimodal Brain Tumor Segmentation Challenge), pp.19-23.

13. T. Zhang, Y. Xia, D. D. Feng, (2012). Clonal Selection Algorithm for Gaussian Mixture Model Based Segmentation of 3D Brain MR Images. Intelligent Science and Intelligent Data Engineering. Pringer Berlin Heidelberg., pp.295302.

14. J. G. Park, C. Lee, (2009). Skull Stripping Based on Region Growing for Magnetic Resonance Brain Images. NeuroImage, vol.47, no.4, pp.1394-1407.

15. K. Somasundaram, T. Kalaiselvi, (2011). Automatic Brain Extraction Methods for T1 Magnetic Resonance Images Using Region Labeling and Morphological Operations. Computers in Biology and Medicine, vol.41, no.8, pp.716-725. 